\title{
Qualitative SERS Analysis of G-Quadruplex DNAs Using Selective Stabilising Ligands
}

\author{
K. Gracie ${ }^{a}$, V. Dhamodharan ${ }^{b}$, P. I. Pradeepkumar ${ }^{b}$, K. Faulds ${ }^{a}$, D. Graham ${ }^{a} *$ \\ ${ }^{a}$ Centre of Molecular Nanometrology, WestChem, Department of Pure and Applied Chemistry, \\ University of Strathclyde, 295 Cathedral Street, Glasgow, G1 1XL, UK \\ ${ }^{b}$ Department of Chemistry, Indian Institute of Technology Bombay, Mumbai 400076, India
}

\begin{abstract}
Nucleic acids are of key biological importance due to their range of functions and ability to form various different structures, with an example of emerging significance being quadruplexes formed by guanine-rich sequences. These guanine rich sequences are found in different regions of the genome such as telomeres, gene promoters and introns and UTRs of mRNAs. Here a new approach has been developed that utilises surface enhanced Raman scattering (SERS) for the detection of the formation of G-quadruplexes. Three G-quadruplex stabilising ligands that each have their own unique SERS response were used in this study and their ability to act as reporters assessed. A SERS response was only obtained from the ligands in the absence of G-quadruplex formation. This resulted in an "on/off" method which was successfully used to qualitatively detect the formation of G-quadruplex using quadruplex-forming sequences such as human telomeric and C-MYC promoter DNAs. The unique SERS spectra of each stabilising ligand offer the potential for use of SERS to study higher order DNA structures. This work shows that the ligands used can act simultaneously as a potential therapeutic stabilising agent and a SERS reporter, therefore allowing the use of SERS as a method of analysis of the formation of G-quadruplex DNAs.
\end{abstract}

\section{Introduction}

G-quadruplexes are guanine rich sequences that are capable of forming stable structures under physiological conditions in vivo. ${ }^{1,2}$ They are composed of G-quartets that are a result of Hoogsteen hydrogen-bonding between guanines, which stack on top of each other producing four-stranded helical structures held together by $\pi-\pi$ interactions. ${ }^{3,4}$ The structure and stability of G-quadruplexes have been investigated using methods such as gel electrophoresis, circular dichroism and UV melting analysis. ${ }^{5}$ The structure and stability of G-quadruplexes are known to be dependent on various factors including the nature of the sequence, flanking nucleotides, loop length and concentration as well as the presence of monovalent cations such as $\mathrm{K}^{+}$and $\mathrm{Na}^{+}{ }^{+6,78}$ The specific cation can have an 
overall effect on the topology of the G-quadruplex. For example, in the presence of $\mathrm{Na}^{+}$, human telomeric DNA sequence, $A G_{3}\left(T_{2} A G_{3}\right)_{3}$, folds into anti-parallel structures, whereas in the presence of $\mathrm{K}^{+}$, TTAGGG repeats can fold into at least five distinct quadruplex structures. ${ }^{9,10}$

Numerous biochemical and structural studies have shown that guanine rich DNA sequences can spontaneously fold into G-quadruplex DNA structures in vitro and more recent studies have shown that quadruplexes have certain functions in vivo. ${ }^{2}$ G-quadruplexes are involved in regulating telomere maintenance, as well as transcription, replication and translation. ${ }^{3}$ Telomeres are nucleoprotein complexes and their main function is to protect the end of eukaryotic chromosomes containing repetitive guanine-rich DNA. ${ }^{1}$ Telomeres can be structurally complex, in somatic cells telomeres are shortened by 50-200 bases after each successive cycle of cell division. This is due to the inability of DNA polymerase to replicate the telomeric DNA strand due to the unavailability of $3^{\prime} \mathrm{OH}$ group at the extreme end of the chromosome (end replication problem). When telomeres are shortened critically, they reach the hayflick limit and cells become senescent and induce apoptotic signals. Nevertheless, telomere length is maintained in $85 \%$ cancers, by the enzyme, telomerase, which is notably absent in somatic cells. The formation of a G-quadruplex structure is an ideal approach to the inhibition of telomerase activity because it is capable of isolating the substrate of telomerase, a single stranded telomeric DNA sequence. ${ }^{11}$

Recently, there has been extensive research on the development of G-quadruplex ligands, particularly ones that target the human telomeric repeat $\left[\mathrm{d}\left(\mathrm{T}_{2} \mathrm{AG}_{3}\right)_{4}\right]$ and would consequently block the action of telomerase. ${ }^{12-14} \mathrm{G}$-quadruplexes have a larger $\pi$-surface area compared to DNA as a result of the four coplanar bases compared to two in duplexes. The majority of small molecules that bind to these structures also have large $\pi$-surfaces in order to maximise the $\pi-\pi$ interactions that will form. ${ }^{15}$ Small organic molecules can lead to cell death by displacing the protective telomeric components or sequestering the substrate needed for telomeric activity. ${ }^{16,17}$ Telomestatin is a natural product that was isolated from the bacterium Streptomyces anulatus and has been shown to be a very potent telomerase inhibitor. ${ }^{18}$

There are several spectroscopic techniques that can be used to investigate the structure and stability of G-quadruplexes, such as UV molecular absorption ${ }^{19}$, circular dichroism (CD) ${ }^{20}$, fluorescence $\left(\right.$ FRET) ${ }^{21}$ and $\mathrm{NMR}^{22}$. UV/CD techniques have the most sensitive instrumental response to the interaction between the G-quadruplex strands, additionally $C D$ can be used to determine if the quadruplex has parallel or anti-parallel configuration. ${ }^{23}$ Raman spectroscopy is an alternative technique which can be used to analyse the structure of macromolecules such as DNA. It was successfully applied to the investigation of the phosphodiester backbone conformation and the 
hydrogen-bonding interactions of quadruplexes. ${ }^{24}$ Miura et al identified specific Raman markers as indicators of the presence of parallel or anti-parallel G-quadruplexes. ${ }^{25}$ Raman spectroscopy is limited by the relatively low cross section of the Raman scattering process compared to other techniques such as fluorescence and absorption spectroscopy. Surface enhanced Raman scattering (SERS) has now become an appealing alternative technique as it overcomes the limitations associated with Raman spectroscopy. ${ }^{26}$ The high sensitivity achieved when using SERS is due to the enhancement of the Raman cross section when the analyte is in close proximity to a metal surface, which is usually in the form of a thin metallic film or a colloidal suspension. There have been several studies involving the direct detection of DNA bases or short DNA sequences utilizing SERS. Kneipp et al applied SERS to the detection of adenine bases, and emphasising the capability of SERS to be used as a method for single molecule detection. ${ }^{27}$ Bell at al successfully used SERS for the label free detection of DNA bases further emphasising the capability of SERS to be used for DNA detection. ${ }^{28}$ The detection of DNA structures has proved to be more difficult due to the enhancement of Raman scattering having a high dependence on the orientation of the DNA with respect to the metallic surface. However, Breuzard et al demonstrated that it is possible to use SERS to analyse DNA structures by using the technique to investigate the stability of G-quadruplexes. ${ }^{29}$ Results revealed that there was a decrease in ethidium bromide SERS signal when it was bound to a quadruplex structure. Rusciano et al then further developed the application of SERS as a method to determine the stability of G-quadruplexes by using direct SERS analysis to gain information on the structure of guanine-rich DNA sequences. ${ }^{30}$ Different G-quadruplex structures were analysed and their relative stabilities were compared. Specific Raman features of G-quadruplex DNAs were identified in the SERS spectra and subsequent analysis on the phosphate backbone conformation and the strength of the Hoogsteen-like hydrogen bonding was performed. ${ }^{30}$ This demonstrated for the first time that SERS can be an extremely useful technique for the analysis of the stabilities of different Gquadruplex structures.

G-quadruplexes can be used as potential therapeutic targets for anti-cancer therapy. ${ }^{31}$ The ligand 2,6-diamidoanthraquinone was shown to stabilise a quadruplex structure and inhibit telomerase activity. ${ }^{32}$ Since then, many classes of ligands have been reported as G-quadruplex stabilising agents, however very few of these have shown specificity towards the targeted quadruplex structure over other DNA topologies. Some ligands have also been shown to be indirect inhibitors of telomerase by displacing the telomere binding proteins that then induce a DNA damage response. ${ }^{33,}{ }^{34}$ Therefore, there has been great interest in developing strong and selective quadruplex binding agents that can detect the presence of quadruplex structures in vivo. ${ }^{17}$ 
Here, we report the use of SERS for the qualitative detection of the formation of G-quadruplex structures. SERS is used as a method to monitor the formation of G-quadruplexes that is based on the unique SERS response given by three G-quadruplex stabilising ligands. When the ligand is added to a solution containing duplex DNA, it remains unbound and free, upon addition of silver nanoparticles aggregation occurs resulting in a strong SERS response. However, when the ligand is added to a solution of G-quadruplex DNAs, the ligand binds strongly to this DNA structure therefore when silver nanoparticles are added; the ligand is no longer free to induce aggregation or a SERS response (Figure 1). The ligands used here are 360A previously reported by Granotier et $a l^{35}$ and the other two ligands, 3AQN and 6AQN, are reported by us and were shown previously to stabilise various quadruplexes (telomeric, C-KIT1, C-KIT2 and C-MYC) selectively over duplex DNA (Figure 2). ${ }^{36}$ For the first time we report the use of SERS as the analysis method due to the ability of the ligands to act as Raman reporters. Various guanine-rich DNA sequences were used in this study, including the human telomeric sequence and the sequence from promoter of C-MYC gene. We have demonstrated the first report of these stabilising ligands being used to analyse the formation of Gquadruplex DNAs over duplex DNA by SERS, which has previously been done using fluorescence based methods.

(a)

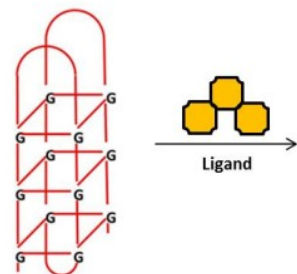

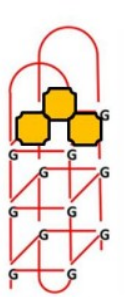

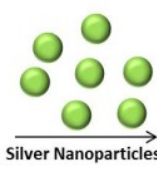

Silver Nanoparticles
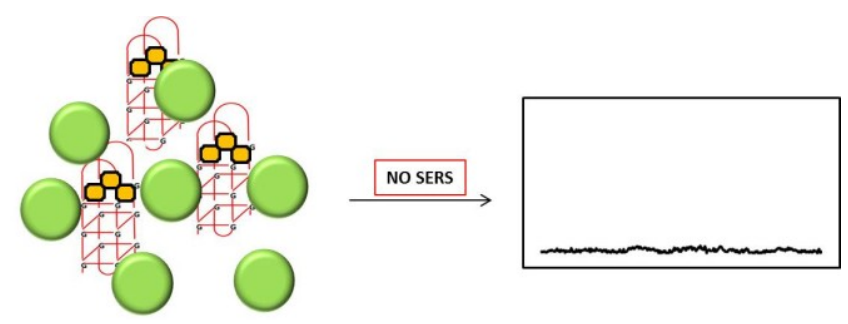

(b)
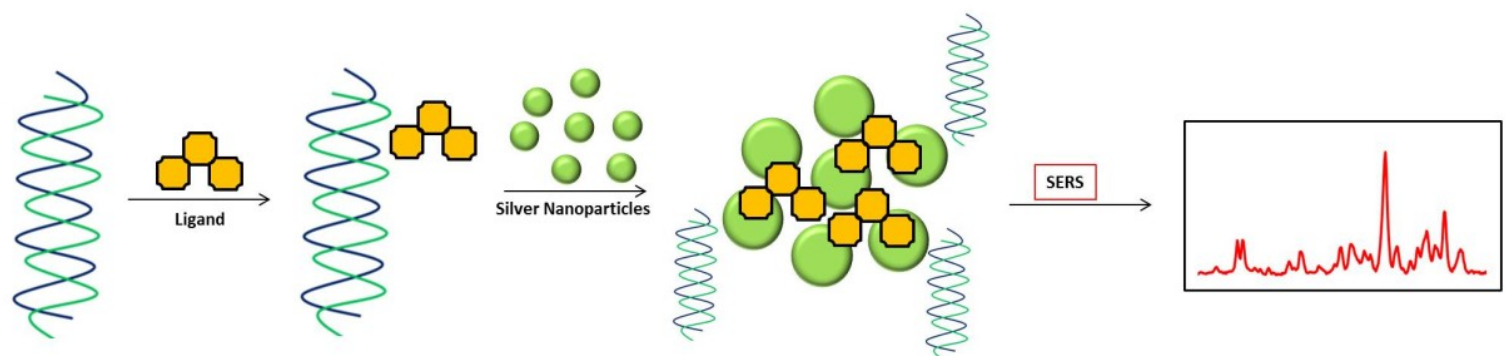

Figure 1 Schematic overview of the qualitative detection of G-quadruplex DNA. (a) When the stabilising ligand is added to a solution containing G-quadruplex DNAs, the ligand strongly binds to the G-quadruplex and when silver nanoparticles are added, the ligand is not free to induce aggregation and a unique SERS response. (b) When the stabilising ligand is added to a solution conatining duplex DNAs, the ligand does not bind to this DNA structure, therefore in the presence of silver nanoparticles, aggreagtion occurs resulting in a SERS response from the ligand. 


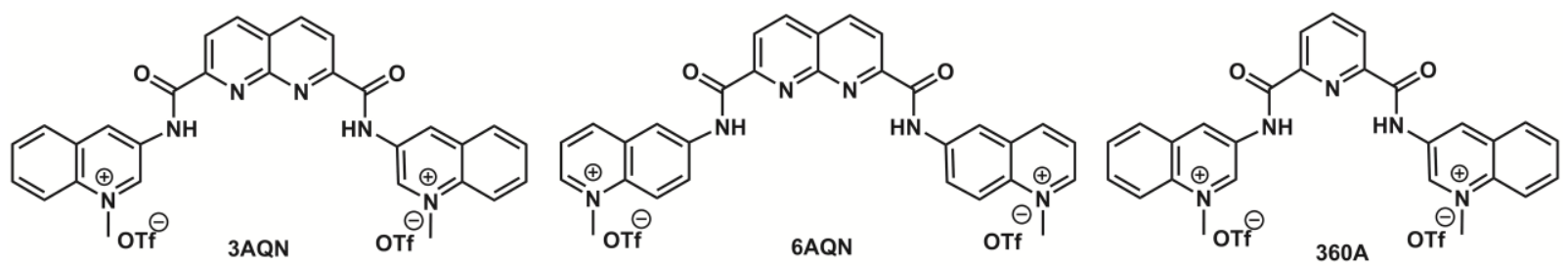

Figure 2 Structures of the three selective G-quadruplex binding ligands. From left: 3AQN (3-amino-quinolinium naphthyridine); 6AQN (6-amino-quinolinium naphthyridine) and 360A (3-amino-quinolinium pyridine).

\section{Materials and Methods}

Ligand Synthesis

The three ligands (3AQN, 6AQN and 360A) used in this study were synthesized as reported previously. ${ }^{36}$

\section{Colloid Synthesis}

Silver nanoparticles were synthesised using a modified Lee and Meisel method. ${ }^{37}$ Silver nitrate $(90 \mathrm{mg})$ was dissolved in distilled water $(500 \mathrm{~mL})$. The solution was heated rapidly to boiling with continuous stirring. Once boiling, an aqueous solution of sodium citrate $(1 \%, 10 \mathrm{~mL})$ was added quickly. The heat was reduced and the solution was left to boil gently for $90 \mathrm{~min}$ with stirring. The colloid was then analysed by UV-vis spectroscopy and the $\lambda_{\max }$ was $403 \mathrm{~nm}$ with the full width halfheight (FWHH) measured to be $124 \mathrm{~nm}$. The concentration of the colloid was calculated to be $0.2 \mathrm{nM}$.

\section{Oligonucleotides}

All DNAs were purchased on a $0.2 \mu$ mole scale with HPLC purification from Eurofins MWG (Ebersberg, Germany).

\begin{tabular}{lll}
\hline Name & Description & Sequence (5' $-\mathbf{3}^{\prime}$ ) \\
\hline Peroxidase Deoxyribozyme & Quadruplex & GTGGGTAGGGCGGGTTGG \\
Human Telomeric DNA & Quadruplex & AGGGTTAGGGTTAGGGTTAGGG \\
C-MYC Promoter & Quadruplex & TGAGGGTGGGTAGGGTGGGTAA \\
Control & $\begin{array}{l}\text { Duplex } \\
\text { (Self complementary) }\end{array}$ & CAATCGGATCGAATTCGATCCGATTG \\
\hline
\end{tabular}

Table 1 Oligonucleotides used in this study. 


\section{SERS Analysis}

SERS analysis was performed using an Avalon Plate Reader (Belfast, UK), with an excitation wavelength of $532 \mathrm{~nm}$. A 96 well plate was placed onto a stage and the instrument's software was used to automatically move the stage so that spectra could be recorded from each well. The accumulation time was $10 \mathrm{~s}$. A single well can hold $300 \mu \mathrm{L}$. Prior to SERS analysis, the ligand $\left(5 \times 10^{-}\right.$

$\left.{ }^{5} \mathrm{M}, 20 \mu \mathrm{L}\right)$ and the specific DNA $\left(5 \times 10^{-5} \mathrm{M}, 20 \mu \mathrm{L}\right)$ were premixed at room temperature for 30 minutes. This mixture was then added to the well that contained citrate reduced silver nanoparticles $(0.2 \mathrm{nM}, 260 \mu \mathrm{L})$. In the studies involving the presence or absence of monovalent cations, either $\mathrm{NaCl}$ $(100 \mathrm{mM}, 20 \mu \mathrm{L})$ or $\mathrm{KCl}(100 \mathrm{mM}, 20 \mu \mathrm{L})$ was used. The samples were then analysed immediately. For peak intensity studies, three replicate samples were scanned 5 times each, with the peak intensities then being averaged to calculate the differences. Spectra were baseline corrected using a multipoint polynomial fit and level and zero mode using Grams software (AI 7.00).

\section{Results and Discussion}

Bisquinolinium ligands such as $360 \mathrm{~A}$ are known to selectively bind and stabilise quadruplex nucleic acids over duplex DNA. ${ }^{38-40}$ Recently we reported two new derivatives of bisquinolinium carboxamide having 1,8-naphthyridine as the central core ${ }^{36}$ (Figure 2). Various biophysical and biochemical studies were used to investigate the interaction between the ligands and different nucleic acids. Circular dichroism (CD) melting assays indicated that ligands can impart higher stabilisation to quadruplex DNA over duplex DNA. Furthermore, CD studies were used to investigate the potential of these ligands to induce quadruplex formation in different DNA sequences. As observed with telomestatin, in the absence of monovalent cations, the ligands induced the formation of a quadruplex structure. A Fluorescent Intercalator Displacement (FID) assay demonstrated the ligands (3AQN, 6AQN and 360A) have high binding affinity and selectivity for quadruplex DNA over duplex DNA. Here, we report the use of surface enhanced Raman scattering (SERS) for the direct detection of the interaction of these ligands with G-quadruplex DNA that does not require the additional use of any other fluorescent agent. This is possible as the ligands themselves give a unique SERS response therefore the interaction between the ligand and DNA can be directly measured. This has potential future implications for the detection of the formation of Gquadruplexes in vivo for use in diagnostic applications. 
Initial experiments were performed to determine if the three ligands gave a SERS response by addition of the ligands directly to a suspension of silver nanoparticles. As can be seen in Figure 3, each ligand had a unique SERS response due to their structural variations. A SERS response was obtained due to the ability of the ligands at the concentration used $(50 \mu \mathrm{M})$ to aggregate the nanoparticles causing the SERS enhancement.

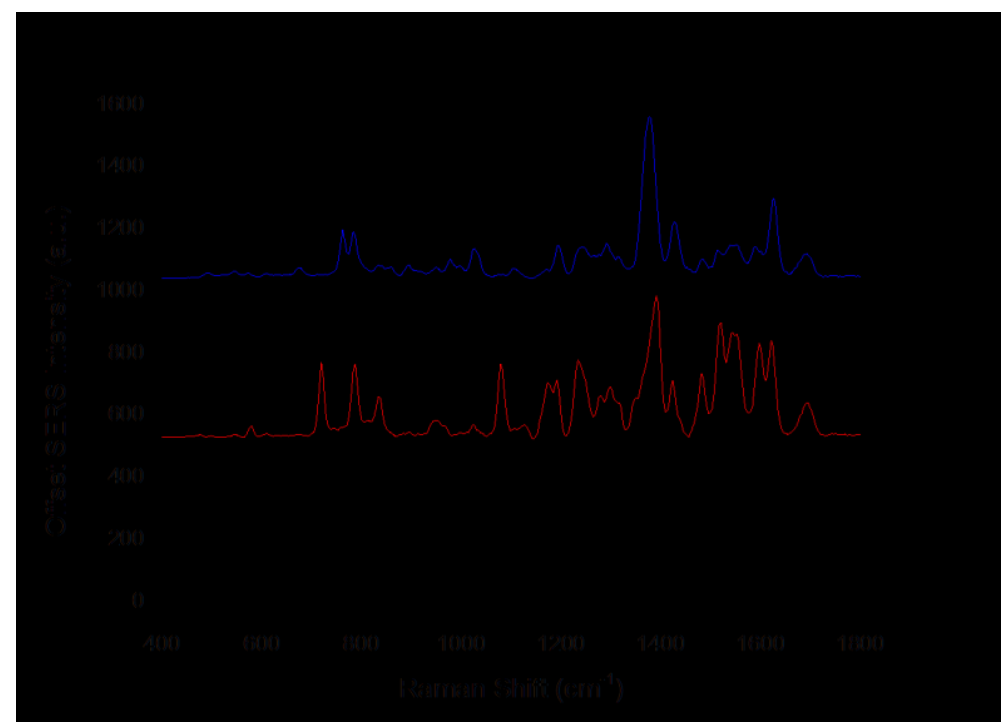

Figure 3 SERS spectra obtained for each stabilising ligand using citrate reduced silver nanoparticles. (a) SERS spectrum of 3AQN, (b) SERS spectrum of 6AQN and (c) SERS spectrum of 360A. Spectra were obtained using $532 \mathrm{~nm}$ laser excitation and an accumulation time of 1 second.

With the knowledge that each of the ligands gave a unique SERS response, they were then incubated for 30 minutes at room temperature with the peroxidase deoxyribozyme quadruplex sequence (Table 1), to study the SERS signal when the ligand was bound to G-quadruplex DNAs. As can be seen in Figure 4, there is a significant reduction in SERS signal when each of the three ligands is bound to the G-quadruplex sequence. This indicates that the ligand was now complexed into the Gquadruplex DNAs, which prevented the ligands from interacting with the surface of the silver nanoparticles, resulting in the reduced SERS intensity observed. 


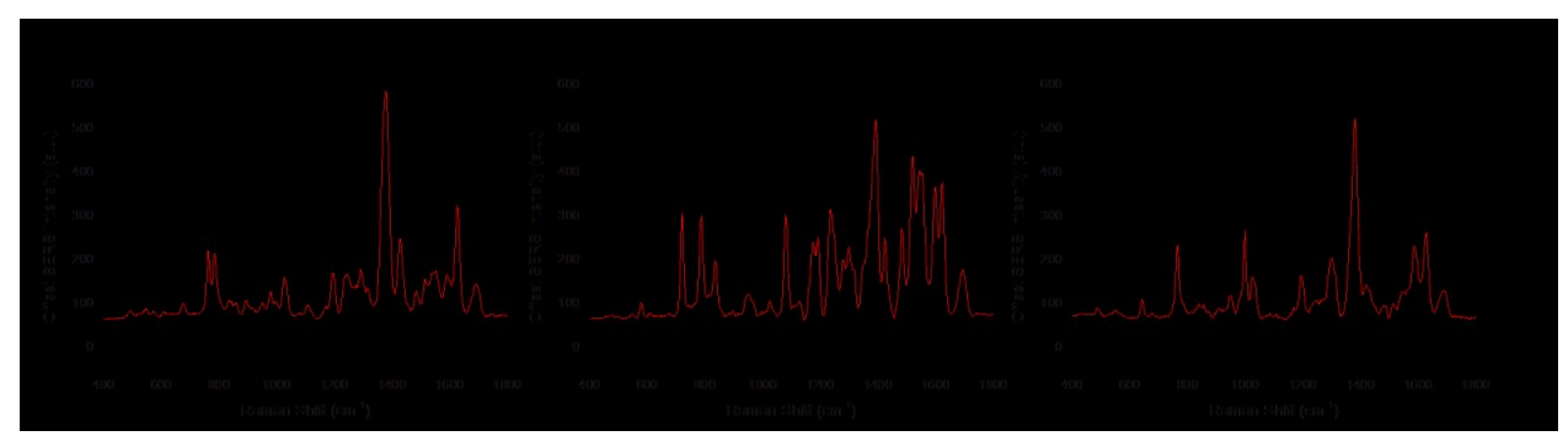

Figure 4 SERS spectra of free ligand (red) and peroxidase deoxyribozyme complex (black): (a) spectra of 3AQN free and complexed to G-quadruplex DNA, (b) spectra of 6AQN free and complexed to G-quadruplex sequence and (c) spectra of $360 \mathrm{~A}$ free and complexed to G-quadruplex sequence. Spectra were obtained using $532 \mathrm{~nm}$ laser excitation and an accumulation time of 1 second.

To demonstrate the specificity of the binding ligands towards G-quadruplexes, the SERS signal was obtained from the interaction of the ligands with a duplex DNA compared to a G-quadruplex structure (Table1), the results are shown in Figure 5. For each ligand, SERS signals were obtained when the duplex control sequence was used in place of the G-quadruplex sequence. This suggests that the ligands do not bind strongly to the duplex allowing them to remain free in solution and to come into close proximity to the surface of the silver nanoparticles resulting in a SERS signal. However, when a G-quadruplex is present a much reduced SERS signal is observed (Figure 1). This confirms the specificity that each of the ligands has towards G-quadruplex DNAs compared to other DNA structures and the strong binding affinity that the ligands have for a G-quadruplex structure. As mentioned previously, the ligands are able to cause nanoparticle aggregation, which means that no aggregating agent was used. If an aggregating agent was added to the SERS mixture, then it would be possible to observe DNA peaks in the spectrum, as well as ligand peaks. Studies have shown that SERS can be used to analyse G-quadruplex and duplex sequences without the use of Raman reporters, however this was performed using a laser excitation frequency of $785 \mathrm{~nm}$ and the addition of an aggregating agents such as magnesium sulphate $\left(\mathrm{MgSO}_{4}\right)$ or spermine. ${ }^{28,41}$ Here the SERS measurements were performed using a laser excitation of $532 \mathrm{~nm}$ in the absence of any aggregating agent. 


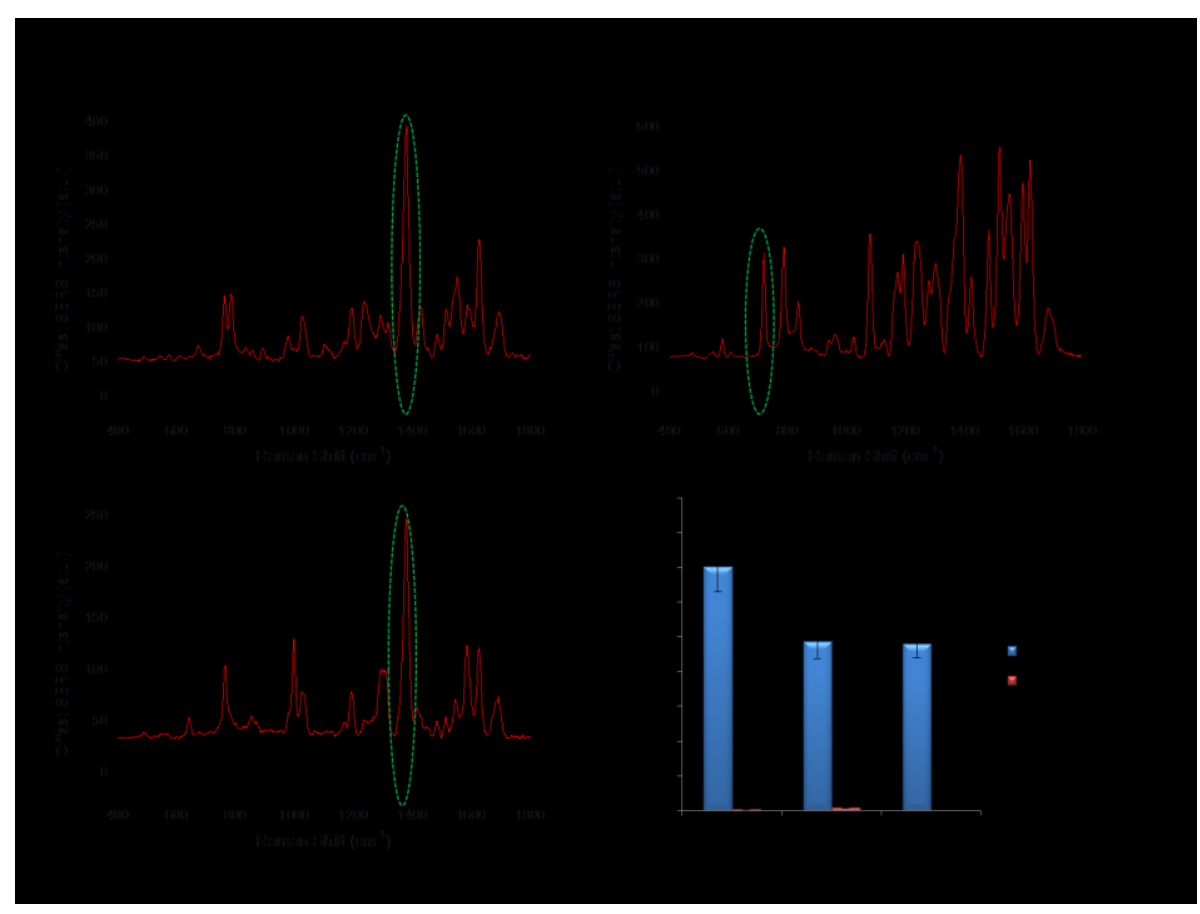

Figure 5 SERS spectra of each ligand binding to the peroxidase deoxyribozyme quadruplex (black) and the spectra obtained when the ligand is in the presence of duplex DNA control sequence (red): (a) spectra of 3AQN binding events; (b) spectra of 6AQN binding events and (c) spectra of 360A binding events. All three ligands and G-quadruplex were used at $50 \mu \mathrm{M}$. The bar chart, (d), is a comparison of the peak intensities of unique peaks of each ligand, highlighted by the green circle. In the spectrum of $3 A Q N$, the peak at $1380 \mathrm{~cm}^{-1}$ was used for comparison studies, the peak at $719 \mathrm{~cm}^{-1}$ in the 6AQN spectrum was chosen and for $360 \mathrm{~A}$ peak intensity studies, the unique peak at $1379 \mathrm{~cm}^{-1}$ was used. Spectra were obtained using $532 \mathrm{~nm}$ laser excitation and an accumulation time of 1 second. All peak intensities were obtained by scanning 3 replicate samples 5 times with an accumulation time of 1 second. Averages are shown and error bars are \pm one standard deviation.

These results were obtained when the ligands and the G-quadruplex DNA in equimolar concentrations $(50 \mu \mathrm{M})$. An investigation was carried out to determine what effect reducing the concentration of G-quadruplex, whilst keeping the concentration of ligand constant, would have on the SERS response. Figure 6 shows the SERS results obtained when the G-quadruplex concentration was reduced from $50 \mu \mathrm{M}$ to $5 \mathrm{nM}$. It can be seen that when the G-quadruplex concentration was reduced to below $1 \mu \mathrm{M}$ a SERS response was obtained due to insufficient G-quadruplex being present to sequester all of the ligand resulting in signal being obtained. This demonstrates that, as expected, the ratio of ligand to G-quadruplex is vital. For example, if the formation of a Gquadruplex was to be monitored using a lower concentration of DNA then a corresponding lower concentration of ligand should be used however, it will be at this point an aggregating agent will be required as the amount of ligand present would not cause the aggregation previously obtained. It can be clearly seen in Figure 6 that by using the correct ratio of DNA to ligand results in a clear, sharp "on to off" signal being obtained upon formation of the G-quadruplex. 


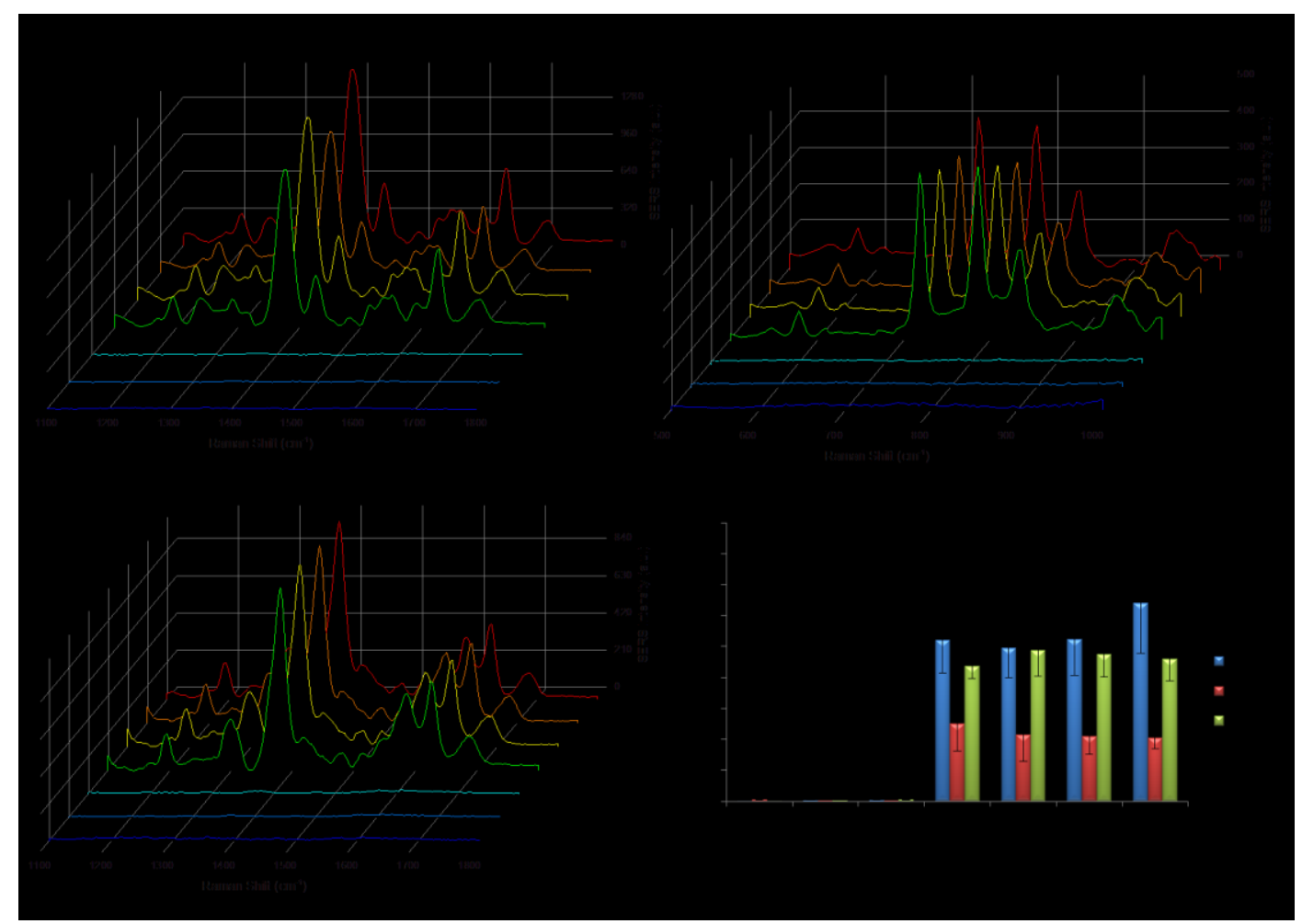

Figure 6 SERS spectra obtained from G-quadruplex concentration studies using each of the three ligands. The Gquadruplex concentration was reduced from $50 \mu \mathrm{M}$ (dark blue), $10 \mu \mathrm{M}$ (light blue), $5 \mu \mathrm{M}$ (turquoise), $0.5 \mu \mathrm{M}$ (green), $50 \mathrm{nM}$ (yellow), $5 \mathrm{nM}$ (orange) and no G-quadruplex present (red). The concentration of the ligands remained constant at $50 \mu \mathrm{M}$. (a) SERS spectra of 3AQN obtained when reducing the G-quadruplex concentration. (b) SERS spectra of 6AQN obtained when decreasing the G-quadruplex concentration. (c) SERS spectra of 360A obtained when decreasing the Gquadruplex concentration. (d) Peak intensity comparisons for each of the three ligands using; $1380 \mathrm{~cm}^{-1}$ for $3 A Q N$ comparisons, $719 \mathrm{~cm}^{-1}$ for $6 A Q N$ comparisons and $1379 \mathrm{~cm}^{-1}$ peak intensity comparisons. Spectra were obtained using $532 \mathrm{~nm}$ laser excitation and an accumulation time of 1 second. All peak intensities were obtained by scanning 3 replicate samples 5 times with an accumulation time of 1 second. Averages are shown and error bars are \pm one standard deviation.

Subsequent studies focussed on the ligand $3 A Q N$ and its behaviour towards various G-quadruplex DNAs and in different salt conditions. Results obtained thus far indicated that in each situation the ligand is bound to the G-quadruplex DNAs, i.e. when the ligand is no longer "free" there is a marked decrease in SERS intensity. For a G-quadruplex structure to form, it is reported that the presence of a monovalent ion, either $\mathrm{K}^{+}$or $\mathrm{Na}^{+}$, is essential. ${ }^{3,5}$ Experiments for this study were originally carried out using either $100 \mathrm{mM} \mathrm{KCl}$ or $100 \mathrm{mM} \mathrm{NaCl}$ to provide these monovalent ions for quadruplex formation. However, it was reported that when using certain stabilising agents such as the ligand $3 A Q N$, neither salt is needed for G-quadruplex formation; the cation presence actually influences the orientation of the quadruplex structure, whether it is parallel or antiparallel. ${ }^{16,36,42}$ This is due to the ability of 3AQN itself, to induce the formation of a G-quadruplex reported by us. ${ }^{36}$ Not only does it allow the formation of the desired structure, it also stabilises the quadruplex, which can be exploited to detect stable quadruplex structures in vivo. ${ }^{17}$ Figure $7(\mathrm{a}-\mathrm{c})$ shows the SERS spectra obtained when $\mathrm{NaCl}$ or $\mathrm{KCl}$ is present and when salt is replaced by water in the reaction mixture and further analysis 
of the intensity of the peak at $1380 \mathrm{~cm}^{-1}$ was performed (Fig 7d). There was a decrease in the absolute SERS intensity when the monovalent ions were absent due to the reduced aggregation of the nanoparticles that would have been caused by the salt, however the discrimination between the double stranded control DNA sequence and the G-quadruplex DNAs was still very significant. SERS signals were only observed when the G-quadruplex DNA was absent; no SERS signals were observed when the ligand was bound to a G-quadruplex sequence, regardless of the salt conditions. This further highlights the potential of using $3 A Q N$ as a stabilising agent for the formation of Gquadruplex DNAs as SERS has shown that it stabilises the G-quadruplex in the absence of monovalent cations.
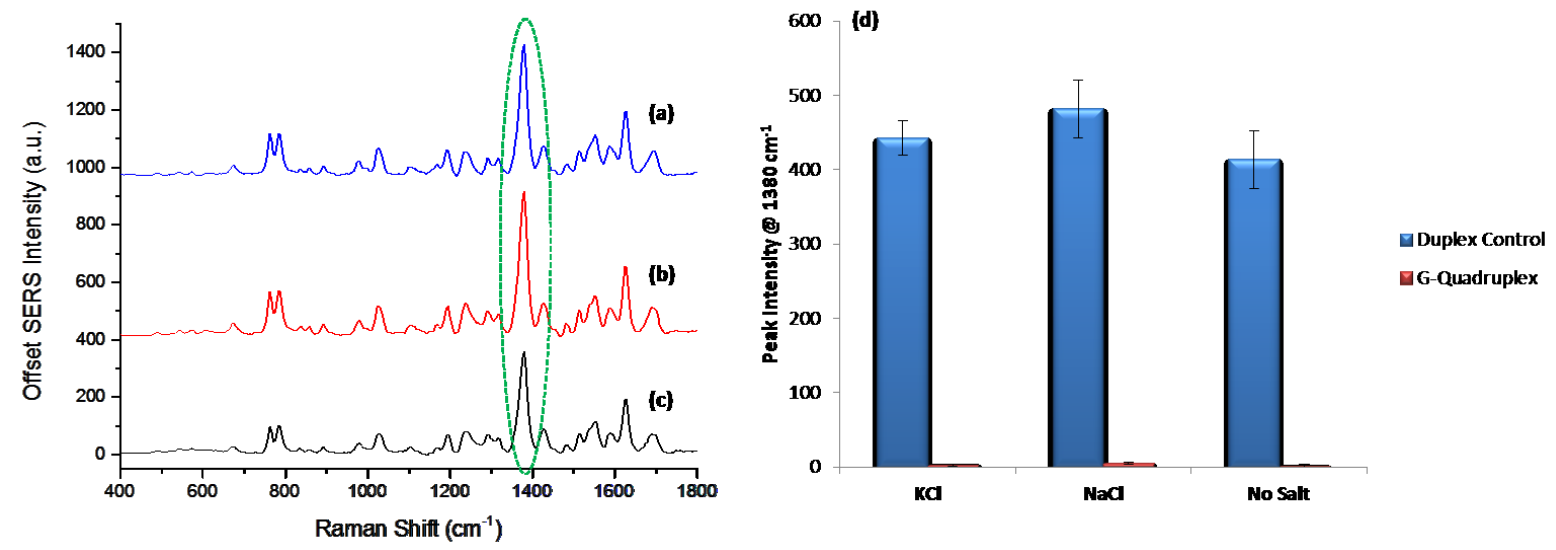

Figure 7 SERS spectra of 3AQN complexed to the duplex DNA control sequence in the presence of (a) $\mathrm{Na}^{+}$ions, (b) $\mathrm{K}^{+}$ions and (c) no monovalent ions. Spectra were also recorded in the different salt conditions when 3AQN was complexed to the peroxidase deoxyribozyme quadruplex sequence. (d) Comparison of peak intensities using the unique peak at $1380 \mathrm{~cm}^{-1}$. Spectra were obtained using $532 \mathrm{~nm}$ laser excitation and an accumulation time of 1 second. All peak intensities were obtained by scanning 3 replicate samples 5 times with an accumulation time of 1 second. Averages are shown and error bars are \pm one standard deviation.

G-quadruplex forming sequences are also found in the promoter regions of oncogenes such as $C$ MYC. The C-MYC oncogene is the most common malfunctioning gene in human cancers; therefore it is an appealing target for anti-cancer therapies. ${ }^{43}$ The human C-MYC gene is highly regulated and any changes in its expression are a crucial point in cancer progression. Detection of these guanine-rich sequences is extremely beneficial to the field of cancer diagnostics. Results shown so far have proved that 3AQN can be used to successfully determine if a G-quadruplex sequence is present or absent. Therefore, studies were carried out to determine if $3 A Q N$ would bind to a G-quadruplex structure present in telomeric or in a proto-oncogene region such as C-MYC. 

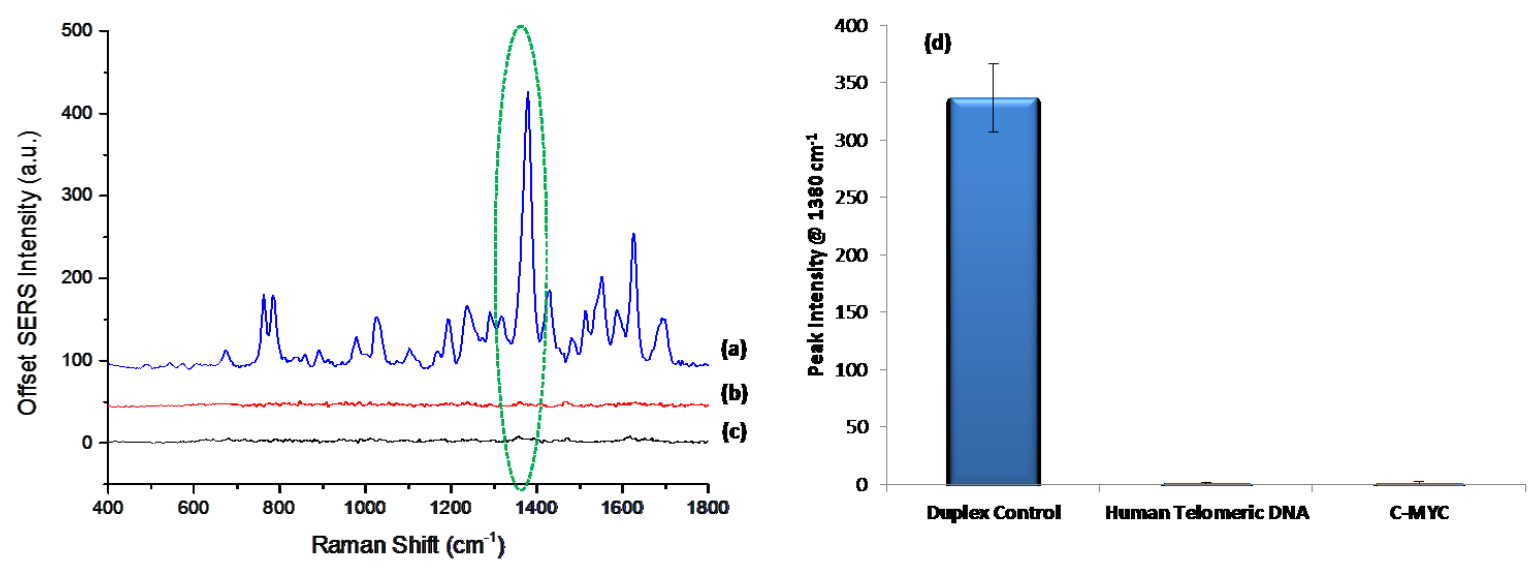

Figure 8 SERS spectra of 3AQN in the presence of (a) control duplex DNA, (b) human telomeric DNA, (c) C-MYC DNA and (d) a comparison of peak intensity at $1380 \mathrm{~cm}^{-1}$. Spectra were obtained using $532 \mathrm{~nm}$ laser excitation and an accumulation time of 1 second. All peak intensities were obtained using the $1380 \mathrm{~cm}^{-1}$ peak by scanning 3 replicate samples 5 times with an accumulation time of 1 second. Averages are shown and error bars are \pm one standard deviation.

Figure $8(\mathrm{a}-\mathrm{c})$ shows that ligand peaks are only observed in the absence of a guanine rich DNA sequence, regardless of whether it is present in a telomeric end or in a proto-oncogene such as $C$ MYC. This demonstrates the strong affinity the ligand 3AQN has for a G-quadruplex topology, regardless of where it is found in a biological system and in the absence of the essential monovalent ions. Figure $8 \mathrm{~d}$ is a comparison of the peak intensities at $1380 \mathrm{~cm}^{-1}$ and as can clearly be seen there is a large discrimination between the ligand in the presence of the duplex control DNA and when the ligand is complexed to G-quadruplex DNAs. These results reaffirm the concept of using SERS and a Gquadruplex binding ligand such as $3 A Q N$ as a specific, efficient and reliable qualitative detection method of telomeric sequences or cancer promoting genes such as C-MYC.

\section{Conclusions}

Detection of the formation of G-quadruplex DNAs found in the peroxidase deoxyribozyme, telomeric region and in the proto-oncogene C-MYC have been demonstrated by a SERS based approach. Studies were carried out involving three quadruplex-binding ligands; 360A, 6AQN and 3AQN. Each produced successful results allowing detection of the formation of G-quadruplexes with high specificity. Further studies were performed using $3 A Q N$ that demonstrated the added stabilising affect offered by this ligand, due to the fact that the monovalent ions usually required for Gquadruplex formation are not needed when using $3 A Q N$, can be directly detected using SERS without adding additional fluorescent agents and even in a salt-deficient condition. This is due to the 
ability of this ligand itself to both induce the formation of the G-quadruplex structure and give a unique SERS response. Since the ligands used here are known to bind/stabilise various quadruplexes, this method may be extended to any quadruplex forming sequences present in the biological landscape. This study also implies that SERS could be used to distinguish a particular quadruplex topology over other quadruplex structures by employing quadruplex-specific ligands. By using SERS, reliable results were obtained in a short time frame without the need for extensive laboratory work and additional assays. The absence of an aggregating agent resulted in the lack of DNA peaks in the SERS spectra, meaning the peaks observed result from the presence of the G-quadruplex stabilising ligands. This is a new and information rich approach to higher order DNA structural analysis and offers significant opportunities for use in studies involving G-quadruplexes as key biological targets.

\section{Acknowledgements}

KF and KG wish to thank the RSC Analytical Trust Fund and EPSRC for funding through the award of DTG funding to KG. DG thanks the Royal Society for support from a Wolfson Research Merit award. P.I.P. thank Council of Scientific and Industrial Research (CSIR), and Department of Atomic EnergyBoard of Research in Nuclear Sciences (BRNS-DAE), Government of India for the research grants. V.D. thanks CSIR the for the fellowship. We also thank Prof. R. Murugavel, IIT Bombay for catalyzing IIT Bombay-Strathclyde collaborations.

\section{References}

1. I. A. Pedroso, L. F. Duarte, G. Yanez, K. Burkewitz and T. M. Fletcher, Biopolymers, 2007, 87, 74-84.

2. E. Y. Lam, D. Beraldi, D. Tannahill and S. Balasubramanian, Nature Communications, 2013, 4, 1796-1796.

3. H. J. Lipps and D. Rhodes, Trends in cell biology, 2009, 19, 414-422.

4. G. N. Parkinson, M. P. H. Lee and S. Neidle, Nature, 2002, 417, 876-880.

5. H.-Q. Yu, D. Miyoshi and N. Sugimoto, Journal of the American Chemical Society, 2006, 128, 15461-15468.

6. Y. Chen and D. Yang, in Current Protocols in Nucleic Acid Chemistry, John Wiley \& Sons, Inc., 2001.

7. S. Burge, G. N. Parkinson, P. Hazel, A. K. Todd and S. Neidle, Nucleic Acids Res., 2006, 34, 5402-5415.

8. N. V. Hud, F. W. Smith, F. A. L. Anet and J. Feigon, Biochemistry, 1996, 35, 15383-15390.

9. B. Heddi and A. T. Phan, Journal of the American Chemical Society, 2011, 133, 9824-9833.

10. A. T. Phan, Febs J., 2010, 277, 1107-1117.

11. M. Y. Kim, M. Gleason-Guzman, E. Izbicka, D. Nishioka and L. H. Hurley, Cancer Res., 2003, 63, 3247-3256. 
12. S. Neidle and G. Parkinson, Nature Reviews Drug Discovery, 2002, 1, 383-393.

13. L. Oganesian and T. M. Bryan, Bioessays, 2007, 29, 155-165.

14. D. J. Patel, A. T. Phan and V. Kuryavyi, Nucleic Acids Res., 2007, 35, 7429-7455.

15. J. L. Huppert, Chemical Society Reviews, 2008, 37, 1375-1384.

16. M. Y. Kim, H. Vankayalapati, S. Kazuo, K. Wierzba and L. H. Hurley, Journal of the American Chemical Society, 2002, 124, 2098-2099.

17. G. Biffi, D. Tannahill, J. McCafferty and S. Balasubramanian, Nature Chemistry, 2013, 5, 182186.

18. K. Shin-ya, K. Wierzba, K.-i. Matsuo, T. Ohtani, Y. Yamada, K. Furihata, Y. Hayakawa and H. Seto, Journal of the American Chemical Society, 2001, 123, 1262-1263.

19. M. Fialova, J. Kypr and M. Vorlickova, Biochemical and Biophysical Research Communications, 2006, 344, 50-54.

20. Y. Xu, Y. Noguchi and H. Sugiyama, Bioorganic \& Medicinal Chemistry, 2006, 14, 5584-5591.

21. F. He, Y. Tang, S. Wang, Y. Li and D. Zhu, Journal of the American Chemical Society, 2005, 127, 12343-12346.

22. O. Y. Fedoroff, M. Salazar, H. Han, V. V. Chemeris, S. M. Kerwin and L. H. Hurley, Biochemistry, 1998, 37, 12367-12374.

23. V. Viglasky, L. Bauer and K. Tluckova, Biochemistry, 2010, 49, 2110-2120.

24. C. Wei, G. Jia, J. Yuan, Z. Feng and C. Li, Biochemistry, 2006, 45, 6681-6691.

25. T. Miura and G. J. Thomas, Biochemistry, 1994, 33, 7848-7856.

26. K. Kneipp, H. Kneipp, I. Itzkan, R. R. Dasari and M. S. Feld, Chemical Reviews, 1999, 99, 29572975.

27. K. Kneipp, H. Kneipp, V. B. Kartha, R. Manoharan, G. Deinum, I. Itzkan, R. R. Dasari and M. S. Feld, Physical Review E, 1998, 57, R6281-R6284.

28. S. E. J. Bell and N. M. S. Sirimuthu, Journal of the American Chemical Society, 2006, 128, 15580-15581.

29. G. Breuzard, J. M. Millot, J. F. Riou and M. Manfait, Analytical Chemistry, 2003, 75, 43054311.

30. G. Rusciano, A. C. De Luca, G. Pesce, A. Sasso, G. Oliviero, J. Amato, N. Borbone, S. D'Errico, V. Piccialli, G. Piccialli and L. Mayol, Analytical Chemistry, 2011, 83, 6849-6855.

31. S. Balasubramanian, L. H. Hurley and S. Neidle, Nature Reviews Drug Discovery, 2011, 10, 261-275.

32. D. Y. Sun, B. Thompson, B. E. Cathers, M. Salazar, S. M. Kerwin, J. O. Trent, T. C. Jenkins, S. Neidle and L. H. Hurley, J. Med. Chem., 1997, 40, 2113-2116.

33. S. Neidle, Febs J., 2010, 277, 1118-1125.

34. S. Balasubramanian and S. Neidle, Curr. Opin. Chem. Biol., 2009, 13, 345-353.

35. C. Granotier, G. Pennarun, L. Riou, F. Hoffschir, L. R. Gauthier, A. De Cian, D. Gomez, E. Mandine, J. F. Riou, J. L. Mergny, P. Mailliet, B. Dutrillaux and F. D. Boussin, Nucleic Acids Res., 2005, 33, 4182-4190.

36. V. Dhamodharan, S. Harikrishna, C. Jagadeeswaran, K. Halder and P. I. Pradeepkumar, J. Org. Chem., 2012, 77, 229-242.

37. P. C. Lee and D. Meisel, Journal of Physical Chemistry, 1982, 86, 3391-3395.

38. J. F. Riou, L. Guittat, P. Mailliet, A. Laoui, E. Renou, O. Petitgenet, F. Mégnin-Chanet, C. Hélène and J. L. Mergny, Proceedings of the National Academy of Sciences, 2002, 99, 26722677.

39. G. Pennarun, C. Granotier, L. R. Gauthier, D. Gomez, F. Hoffschir, E. Mandine, J. F. Riou, J. L. Mergny, P. Mailliet and F. D. Boussin, Oncogene, 2005, 24, 2917-2928.

40. A. De Cian, E. DeLemos, J.-L. Mergny, M.-P. Teulade-Fichou and D. Monchaud, Journal of the American Chemical Society, 2007, 129, 1856-1857.

41. G. Rusciano, A. C. De Luca, G. Pesce, A. Sasso, G. Oliviero, J. Amato, N. Borbone, S. D'Errico, V. Piccialli, G. Piccialli and L. Mayol, Analytical Chemistry, 2011, 83, 6849-6855. 
42. E. Izbicka, R. T. Wheelhouse, E. Raymond, K. K. Davidson, R. A. Lawrence, D. Y. Sun, B. E. Windle, L. H. Hurley and D. D. Von Hoff, Cancer Res., 1999, 59, 639-644.

43. A. Siddiqui-Jain, C. L. Grand, D. J. Bearss and L. H. Hurley, Proceedings of the National Academy of Sciences, 2002, 99, 11593-11598. 